\title{
The influence on Dobutamine docking of blood flow around the sinoatrial node
}

\author{
A. K. Macpherson ${ }^{1}$, S. Neti ${ }^{1}$, M. Averbach ${ }^{2}$, P. A. Macpherson ${ }^{3}$, \\ C. Chu Takositkanon ${ }^{1} \&$ M. Chaney ${ }^{4}$ \\ ${ }^{1}$ Institute of Biomedical Engineering and Mathematical Biology, \\ Lehigh University, USA \\ ${ }^{2}$ Division of Cardiology, St Lukes Hospital, USA \\ ${ }^{3}$ Department of Applied Technology, Rogers State University, USA \\ ${ }^{4}$ Department of Surgery, College of Physicians \& Surgeons, \\ Columbia University, USA
}

\begin{abstract}
Traditionally, the docking of drugs onto cell receptors has been considered to occur from the capillaries into the interstitial fluid and thence to the cell receptor. In patients that are unable to exercise, pharmacologic stress testing is performed, either with vasodilatory agents (e.g. adenosine) or Dobutamine, which is a proinotropic and chronotropic drug. This drug docks with the sinoatrial node (SAN) in the upper right atrium of the heart near the superior vena cava. Due to its unique location it is exposed to both the blood flow coming from the right ventricle as well as the flow in the interstitial fluid. The SAN in the human has a surface area of approximately $4.6 \mathrm{~mm}^{2}$ and $0.1-0.2 \mathrm{~mm}$ thick and hence has a large surface area to volume ratio. The flow on the outside was simulated by a multiscale model consisting of a continuum flow, a Monte Carlo region and at the inner surface a molecular dynamics model. The simulation of the interstitial fluid contains more unknowns than the external flow. The SAN is relatively thin and thus it does not contain as many capillaries as would be found in normal tissue. The drug molecular dimensions are such that the Dobutamine can readily pass through the spaces between the capillary molecules, usually approximately 40 microns. Due to the number of unknowns involved with the capillary solution a continuum solution involving a diffusion coefficient based on the Lebas molecular volume was undertaken to obtain the order of magnitude of the
\end{abstract}


docking. It was found that the number of drug molecules docking on the surface was much greater than the number docking through the interstitial fluid.

Keywords: sinoatrial, Dobutamine, drug docking, bloodflow, drug diffusion.

\section{Introduction}

One method to evaluate for underlying coronary artery disease is to perform stress testing. Often, myocardial stress is achieved by the patient walking on a treadmill or riding a stationary bicycle while being monitored. In patients that are unable to exercise, pharmacologic stress testing is performed, either with vasodilatory agents (e.g. adenosine) or Dobutamine, which is a pro-inotropic and chronotropic drug. Dobutamine (the drug) is injected in the patients arm and is absorbed by the sinoatrial nodes (SA) in the right ventricle. There are receptors on the SA onto which the drug docks. The docking process involves certain atoms on the drug interacting with appropriate atoms in receptor.

There are three possible mechanisms for the drug to affect the membrane, figure 1 [1]. One is by direct docking with the receptor (a), the second is by entering the membrane and then diffusing through the membrane to dock with
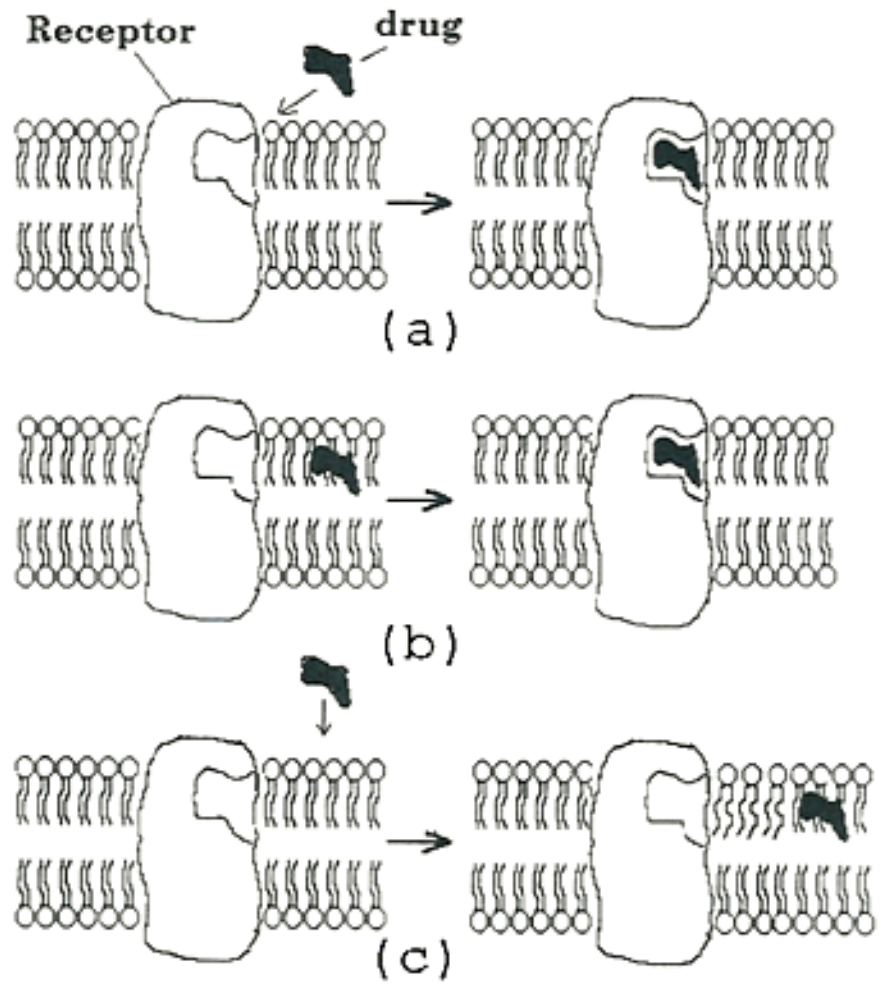

Figure 1: $\quad$ Mechanisms for the drug affect on the membrane. 
the receptor (b). The third is by interacting directly with the membrane. Probably all three mechanisms are involved. However for the present work only the direct action is considered. The SA are the pace makers of the heart and the effect of the Dobutamine is to increase the heart rate. In undertaking an analysis of the effects of the drug it is important to know how much of the drug that is injected is absorbed by the cells. The SA nodes are located at the entrance to the superior vena cava past which the blood flows to the left ventricle. Blood also flows through the interior of the SA via the capillary network. In the present case the ratio of surface area to volume ratio is much larger than in most drug docking situations. Thus it would appear possible that a larger percentage of the drug could be absorbed at the surface as opposed to the usual mechanism by diffusion from the capillary bed. The present paper examines this proposition.

\section{Method of calculation}

Probably the earliest experimental work on Dobutamine docking on beta 1 sites is [13]. Here experiments were undertaken on dogs and it was found that the main effect was 'direct action on beta 1 cardiac receptors'. The effect on beta 2 and alpha sites was slight. The same view has been confirmed many times since and generally persists today.

The Chemical Computing Group's MOE molecular modeling software (Montreal, Quebec, http://www.chemcomp.com) was used for protein construction, visualization and docking calculations. The structure of the human beta 1 adrenergic receptor was based on sequence homology with the known Xray structure of turkey beta 1 receptor, complexed with Cyanopindolol (2VT4, Protein Data Bank). The FASTA routine within Biology WorkBench 3.2 (http://workbench.sdsc.edu) was used for sequence alignment and revealed an E score $=1.8 \mathrm{e}-65$, and pair-wise identity of $77.5 \%$ (ungapped), over 345 amino acid residues. The final structure of the complex was achieved by molecular dynamics simulation and minimization using the Merck Molecular Force Field (MMFF94x) and parameter set, as supplied within MOE program. Specifically a non-bonded cut-off was set at 12.0 Angstoms. Solvation effects were incorporated using the Born implicit model. As it does not appear that the docking of Dobutamine has been studied experimentally a probable binding site was calculated. The Dobutamine structure was built and minimized. It was positioned initially by superimposing it with the Cyanopindolol molecule as observed complexed within the turkey beta 1 structure.

An additional and supporting approach for determining the potential Dobutamine site was guided by using the Alpha Site Finder methodology [14], a feature of the Chemical Computing Group's MOE molecular modeling software. The algorithm calculates and displays potential regions of tight atomic packing on a protein surface. Both methods were in agreement and established a starting point for docking studies using the MOE Flex algorithm. The best binding site was based on a number of scoring functions (AEC scoring, Affinity DG scoring, HB scoring and London DG scoring (http.//chemcom.com for detailed definition). The result of our docking study based on the best score is shown in 
figure 2. The appropriate amino acid sites on the receptor are shown, which indicate a tightly closed and energetically bound ligand. It be seen that there are five possible residue sites on the Beta 1 receptor that are in close contact and contribute to its binding of Dobutamine. Ser 212 and Ser 215 form H-bonds with the meta and the ligand's para-hydroxyls (1.6 A and 1.8 A, respectively). An amino salt- bridge interaction is formed with Asp 121 (1.2 A), along with an Hbond to Asn 329 (1.5 A). The Gly 98 backbone carbonyl is observed to form an H-bond with Dobutamine's other para-hydoxyl group (1.5 A). Also contributing to the stabilization of Dobutamine binding are the hydrophobic and aromatic residues that form hydrophobic and pi-stacking interactions with the ligand's aromatic system.
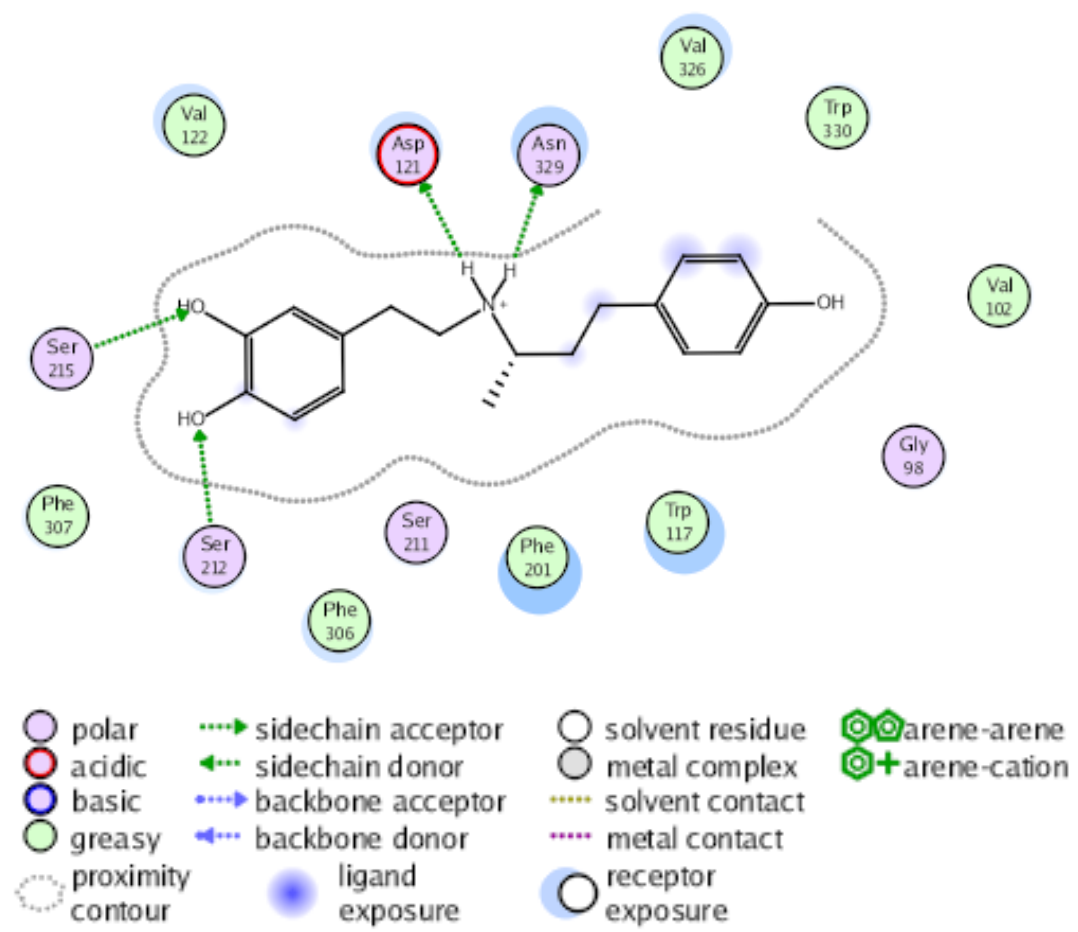

Figure 2: A Two-Dimensional Beta 1 - Dobutamine contact map. Close contacts (less than 3.5 Ang) are displayed indicating those Beta 1 residues that are responsible for binding Dobutamine. The legend indicates the type of interaction, residue properties, ligand and receptor exposure, as well as the proximity countour. Residue numbers refer to human Beta 1.

The external blood flow calculations were undertaken using a multiscale model [5] which was modified for the present calculations. The flow in the region most distant from the surface was a continuum region, the interaction at 
the blood cell size level is a Monte Carlo process and the interactions with the receptors are undertaken using a direct simulation method known as molecular dynamics.

Continuum Scale: As details on neither the dimensions of the right atrium nor the blood flow velocity were available the continuum level solution could not be undertaken. Thus average values were chosen from [6]. The value used was $7.0 \mathrm{x}$ $10^{9} \AA / \mathrm{sec}$.

Blood cell scale: The Monte Carlo method has been described previously in [5] so it will only be briefly reviewed here. The Monte Carlo region of interest $12000 \AA$ x $4000 \AA$ x $3000 \AA$ was divided into 100 cells. The method involves separating the motion into a collisional exchange of information and a convective information exchange. The blood is considered to be composed of water, erythrocyte, albumin, and Dobutamine. As the medium is a liquid then the collisional information was undertaken using the Landau equation.

The initial assignment of particle positions is random in the three directions. The velocities of the particles were set with a Maxwellian distribution appropriate to the temperature

The solution starts by taking a Monte Carlo fraction of the molecules. In the present case the fraction was $1 / 20$. The change of velocity was undertaken using the Landau equation which in the test particle form below has been described as a generalized diffusion equation in velocity space [6]. Expressed in a nondimensional form it becomes

$$
\partial \phi_{\tau}=\partial_{v_{r}}\left(-F_{r}+0.5 \partial_{s} T_{r s}\right) \phi
$$

where $\phi$ is the velocity distribution, the $v_{r}$ differentiation is with respect to the non-dimensional velocity $\mathrm{v} / 2 \mathrm{kT}$, subscript $\tau$ is differentiation with respect to the non-dimensional time defined below.

$$
\begin{gathered}
\boldsymbol{F}_{r}=-8 \boldsymbol{v}^{-1} \boldsymbol{G}(\boldsymbol{v}) \boldsymbol{v}_{r} \\
\boldsymbol{T}_{r s}=2 \boldsymbol{v}^{-1} \boldsymbol{H}(\boldsymbol{v}) \delta_{r s}+2 v^{-3} E(v) v_{r} v_{s}
\end{gathered}
$$

and $\mathrm{H}, \mathrm{G}$ and $\mathrm{E}$ are tabulated [6]. The non-dimensional time [7] is

$$
\boldsymbol{t}=\frac{\boldsymbol{\beta}^{3 / 2} \boldsymbol{B n}}{\boldsymbol{m}^{1 / 2}} \tau
$$

where $\boldsymbol{m}$ is the mass, $\boldsymbol{n}$ the number density, $\boldsymbol{\beta}=1 / \mathrm{kT}$ and $\boldsymbol{B}$ is defined as

$$
\boldsymbol{B}=8 \pi^{5} \int_{0}^{\boldsymbol{l}_{m}} \boldsymbol{l}^{3} V_{\boldsymbol{l}} \boldsymbol{d l}
$$

The force on a particle will consist of a drag due to $G(v)$ and a random force due to $H(v)$. The interaction time scale is as defined in equation (5). The convective step is then implemented. This is achieved by choosing a short length 
of time $\Delta \mathrm{T}$. The particles then move with the velocity $\mathbf{v}$ attained at the end of time $\Delta \mathrm{T}$ for a distance $\mathbf{v} \Delta \mathrm{T}$. New cells are then formed and the process repeated. The boundary conditions as described above are applied at the end of each time step $\Delta \mathrm{T}$. The value of $\Delta \mathrm{T}$ was determined as follows [8]. Within a cell containing $\mathrm{N}$ particles the particle with the largest total interaction cross section $\sigma_{\mathrm{i}}$ is chosen for collision where

$$
\sigma_{i}=\sum_{j=1}^{N} \frac{\left|c_{i}-c_{j}\right|}{c_{i}} \sigma_{o i j}
$$

The cross section is very difficult to calculate in the present case as the particles are so large. Thus two possible interactions were considered. In one case the particles were considered to carry a charge and the collision cross section $\sigma_{\mathrm{oij}}$ is given in terms of the deflection angle $\chi_{\mathrm{m}}$. In the other case, the particles were considered to be hard spheres. The two cases were compared to judge the importance of the cross sectional approximation. The procedure then continues by choosing two colliding particles and time $t$ calculated by

$$
\mathrm{t}_{\mathrm{i}}=-\xi^{*}(\mathrm{n}) /\left(\sigma_{\mathrm{i}} \mathrm{N}\right)
$$

where $\xi$ is a random number between 0 and $1, \mathrm{n}$ is the number of molecules in the cell, $\mathrm{N}$ is the number density. This process is repeated for all cells.

The geometry for the calculation of the diffusion of the Dobutamine in the sinus node is complex as shown in figure 3 from [9]. The arrows point to capillaries. The length of the centre arrow is approximately $50 \mu \mathrm{m}$ long. The distance between the capillaries are then $48 \mu \mathrm{m}$ and $81 \mu \mathrm{m}$. An accurate calculation of a docking process would require detailed knowledge concerning the cell structure, the location of the interstitial fluid, the capillary lengths, the number of capillaries normally active etc., within the limits discussed below the diffusion equation

$$
\frac{\partial C_{N}}{\partial t}=D \frac{\partial^{2} C_{N}}{\partial^{2} x}
$$

can be approximately solved. Although the diffusion process is three dimensional [5], due to the uncertainties in the present case only a one dimensional solution will be considered.

The molecular dynamics region was the region above the surface and below the Monte Carlo region. The beta 1 receptor molecule rises approximately $50 \AA$ above the cell surface. Thus the lower surface of the Monte Carlo region was placed at $57 \AA$ above the cell surface. If a Dobutamine molecule entered the molecular dynamics region it was allowed to proceed at its current velocity to the cell surface. The density of beta 1 receptors of beta 1 receptors was obtained from [10] as $7.7 \mathrm{pmol} / \mathrm{mL}$. Assuming that $30 \%$ of the receptors would be activated at a given time a random number was generated and if it was greater than the probability of hitting a receptor a collision was considered to occur. An 
arbitrary impact parameter was chosen for the Dobutamine molecule as well as an arbitrary rotational angle. The molecule was then the allowed to proceed through the molecular dynamics region till it intercepted the receptor. If the appropriate atoms on the Dobutamine were within $3 \AA$ of a docking site, as shown in figure 2 , then a docking was considered to occur. At this time the receptor was removed from the cell as thus the density of receptors in the cell was reduced. New Dobutamine molecule was introduced at the midpoint of the region of interest.

\section{Results}

The external flow was calculated as described above. Due to the small number of Dobutamine molecules in the blood it is impossible to perform the calculations using the full number of water and erythrocyte molecules. This arises as with a Monte Carlo fraction of 20 there are only two Dobutamine molecules in the region of interest. The number of albumin molecules was set at the correct value. However even using the Monte Carlo fraction of 20 the number of water and erythrocyte molecules would be so large that only a super computer using parallel processing could undertake such a calculation The code was run with the different numbers of water and erythrocyte molecules and it was found that provided the numbers were greater than 50 there did not appear to be any significant changes in the results. In the present calculations the number of water and erythrocyte molecules were set at 500 each. The result of the calculations is shown in table 1.

Table 1: $\quad$ External flow docking of Dobutamine with Beta 1 receptors.

\begin{tabular}{|c|c|c|}
\hline $\begin{array}{c}\text { Number of } \\
\text { Dobutamine } \\
\text { molecules } \\
\text { docking/ } \mu \mathrm{m}^{2}\end{array}$ & $\begin{array}{c}\text { Time } \\
\text { lonic } \\
\text { Cross } \\
\text { Section } \\
\text { Secs. }\end{array}$ & $\begin{array}{c}\text { Time Hard } \\
\text { Sphere Cross } \\
\text { Section Secs }\end{array}$ \\
\hline 2000 & .03382 & 0.0139 \\
\hline 4000 & .07355 & 0.0515 \\
\hline 6000 & .09979 & 0.1148 \\
\hline
\end{tabular}

It can be seen that the assumptions made about the collision cross section is relatively unimportant. This arises because it is only used to calculate the time advance and not the collisional change of velocity.

As described above the docking within the cells is very complex. There does not appear to be experimental values for the diffusion coefficient (D) for Dobutamine. One method of estimating D is from [11]

$$
D=k \frac{1.474 \times 10^{-15}}{\mu^{1.14} V_{B}^{0.589}}
$$


where $\mathrm{D}$ is the diffusivity in $\mathrm{m}^{2} / \mathrm{sec}, \mu$ is the viscosity in $\mathrm{kg} / \mathrm{m}$.sec and $\mathrm{V}_{\mathrm{B}}$ is the LeBas molar volume in $\mathrm{m}^{3} / \mathrm{mol}$. Taking the solvent as water the viscosity is $1.08 \mathrm{e}-3 \mathrm{~kg} / \mathrm{m} . \mathrm{sec}, \mathrm{k}=1$ and the Le Bas volume is $1.053 \mathrm{e}-2$ giving a diffusivity of $3.42 \times 10^{-10} \mathrm{~m}^{2} / \mathrm{sec}$. The diffusion coefficient can also be estimated from the Stokes - Einstein relation $\mathrm{D}=\mathrm{kT} /(6 \pi v \mathrm{r})$. This gives the value of $\mathrm{D}$ as $3.90 \times 10^{-10}$ $\mathrm{m}^{2} / \mathrm{sec}$ for diffusion in water. A value of $3.5 \times 10^{-10} \mathrm{~m}^{2} / \mathrm{sec}$ will be used.

Based on figure 3 the solution of equation (8) would not be very accurate. However an order of magnitude for the time involved can be obtained from an approximation of equation (8).

$$
\mathrm{t}=\lambda^{2} / \mathrm{D}
$$

where $\lambda$ is a typical diffusion length scale. The diffusion length that is significant is the distance from a capillary to a cell. Considering the dimensions discussed above a length of $25 \mu \mathrm{m}$ would be appropriate. Thus an order of magnitude calculation of the time for diffusion can be obtained as $0.18 \mathrm{sec}$. The docking calculation would then increase the time due to the availability of receptors and the orientation of the drug as described for the external flow. From figures 2 and 3 [9] the dimensions of the nodal cells and the capillaries range between $4 \mu \mathrm{mi}$ and $8 \mu \mathrm{m}$. Thus as well as interstitial fluid there is considerable tissue such as mitochondrial cells. In this case the viscosity may be an order of magnitude greater [12] giving a diffusivity of $0.25 \times 10^{-10} \mathrm{~m}^{2} / \mathrm{sec}$. The thickness of the sinus nodes is approximately $200 \mu \mathrm{m}$. Thus there are approximately 5 layers of cells through the nodes.

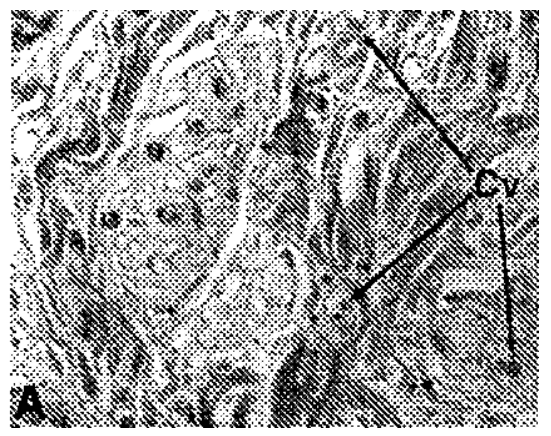

Figure 3: $\quad$ Sinus node histologic section from figure 1 [9].

\section{Conclusions}

Comparing equation 9 and table 1 the docking of Dobutamine on the sinus nodes mainly occurs on the blood side of the nodes. It would be expected that the drug would be mainly absorbed within 1 second of the Dobutamine being distributed around the body. The release time of the Dobutamine from the receptor is known. In order that there remain sufficient receptors available for the 
Dobutamine to dock with, then a release time of less than half a second would appear to be conservative. Thus the present values for the docking time may be reasonable.

This result arises because of the high blood flow past the nodes. A similar situation likely occurs in the ventricles although to a lesser degree. In most situations diffusion is likely to be the principal mechanism.

\section{References}

[1] Mavromoustakos T, Daliani I and Matsoukas 1999 The application of biophysical methods to study Drug Discovery and Design: Medical Aspects Ed J. Matsoukas and T. Mavromoustakos, IOS press, Amsterdam, 13-24.

[2] Goodsell, D.S. and Olson, AJ. (1990) "Automated Docking of Substrates to Proteins by Simulated Annealing”. Proteins: Structure, Function, and Genetics 8:195-202.

[3] Morris, G.M., Goodsell, D.S., Huey, R and Olson, AJ. (1996) “Distributed Automated Docking of Flexible Ligands to Proteins: Parallel Applications of AutoDock 2.4”. J. Computer-Aided Molecular Design 10:293-304.

[4] Morris, G.M., Goodsell, D.S., Halliday, RS, Huey, R, Hart, W.E., Belew, RK., Olson, AJ. (1998) "Automated docking using Lamarckian genetic algorithm and an empirical binding free energy function.” J. Compo Chem. 19:1639-1662.

[5] Macpherson AK and Neti S 2001 "A rapid procedure for initial drug evaluation", Phys. In Med. and Biol.46,6

[6] Sloth E, Kruse M, Houlind KC, Petersen EM, Hasenkam JM, "The impact of ischemic heart disease on main pulmonary flow blood flow patterns : a comparison between magnetic resonance phase velocity mapping and transesophagel colour Doppler" Cardiovascular Research 37,377-385,1997.

[7] Chandrasekhar, S. "Principles of Stellar Dynamics", Uni. of Chicago Press, Chicago, 1942.

[8] Ruth, D.W. 1972 "A Monte Carlo simulation of the impulsively started piston problem, M.S. Thesis, University of Manitoba, Dept. of Mech. Eng., Winnipeg, Canada

[9] Balescu R. Equilibrium and Non Equilibrium Statistical Mechanics, John Wiley, New York, 1990.

[10] Hurlé A, Sánchez-Quintana D, Ho S.Y., Bernabeu E, Murillo M, Climent V Capillary Supply to the sinus Node in Subjects with Long-Term Atrial Fibrillation, The annals of thoracic surgery,89, 1, 38-43, 2010.

[11] Tsukamoto1.T et al 'Decreased Myocardial b-Adrenergic Receptor Density in Relation to Increased Sympathetic Tone in Patients with Nonischemic Cardiomyopathy ",The Journal of Nuclear Medicine, 48, 11, Nov 2007 177182

[12] Lyman, W.J., Reehl, W.F., Rosenblatt, D.H. (eds.) (1990) Handbook of chemical property estimation methods, American Chemical Society, Washington DC, USA. 
[13] Meulemans A, Paycha F, Hannoun P, Vulpillat M, Measurement and Clinical and Pharmacokinetic Implications of Diffusion Coefficients of Antibiotics in Tissues, Antimicrobial Agents and Chemotherapy, Aug 1989, 1286-1290.

[14] Tuttle RR, Mills J. Dobutamine: development of a new catecholamine to selectively increase cardiac contractility. Circ Res. 1975 Jan;36(1):185-96 